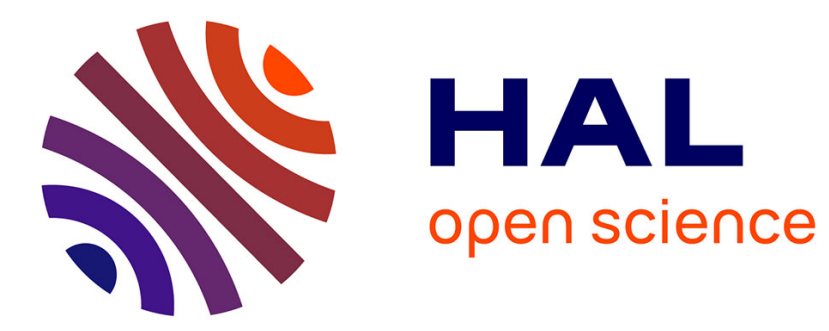

\title{
Time-Frequency Characterization using Instantaneous Moment Concept: Theory and Applications
}

Cedric Cornu, Cornel Ioana, André Quinquis, Srdjan Stankovic

\section{To cite this version:}

Cedric Cornu, Cornel Ioana, André Quinquis, Srdjan Stankovic. Time-Frequency Characterization using Instantaneous Moment Concept: Theory and Applications. IEEE International Symposium on Industrial Electronics, Jul 2006, Montreal, Canada. pp.559-564, 10.1109/ISIE.2006.295519 . hal00349542

\section{HAL Id: hal-00349542 \\ https://hal.science/hal-00349542}

Submitted on 31 Dec 2008

HAL is a multi-disciplinary open access archive for the deposit and dissemination of scientific research documents, whether they are published or not. The documents may come from teaching and research institutions in France or abroad, or from public or private research centers.
L'archive ouverte pluridisciplinaire HAL, est destinée au dépôt et à la diffusion de documents scientifiques de niveau recherche, publiés ou non, émanant des établissements d'enseignement et de recherche français ou étrangers, des laboratoires publics ou privés. 


\title{
Time-Frequency Characterization using Instantaneous Moment Concept : Theory and Applications
}

\author{
Cédric CORNU*, Cornel IOANA*, André QUINQUIS*, Srdjan STANKOVIC** \\ * : ENSIETA, E3I2 Laboratory, 2 rue François Verny, Brest - France \\ E-mails : [cornuce,ioanaco, quinquis] @ensieta.fr \\ ** : University of Montenegro, Podgorica - MONTENEGRO \\ E-mail : srdjan@cg.ac.yu
}

\begin{abstract}
The instantaneous frequency law (IFL) is a very important element when the physical parameters of the corresponding signal have to be evaluated. Blind equalisation, modulation recognition and mechanical diagnostic are just three domains where the non-stationarity behavior of the signal imposes the IFL estimation. Generally, an IFL is composed by slowly varying time-frequency structures separated by fast transitions which could be considered as phase discontinuities. Digital phase modulations or signals propagated trough a multipath channel are typical examples of IFLs having fast transient parts. The common methods employed for these operations are wavelet transform and Cohen's class time-frequency representation, respectively.

In this paper we propose an alternative based on the instantaneous moments. By an appropriate choice of the moment order and lags it is possible to accurately estimate the both transient and slowly time-frequency parts. While this method uses one dimensional data and, thanks to its recursive structure, it is well suited for real time applications. Therefore, its real-time implementation on TMS320C6x structure is described. On the other hand, few examples on realistic data will show the practical interest for this method.
\end{abstract}

\section{INTRODUCTION}

Analysis of the signals characterized by complex timefrequency behaviour is a challenging topic, due to the richness of the information carried by the IFL. In a large number of applications the analysis of the time-frequency (T-F) content provides an efficient solution to the problems arising in these fields [1]. The signals associated to real applications are generally characterized by many time-frequency structures usually considered as slowly varying parts. Connections between these stationary parts are often subject to fast transitions whose estimation can be of great iterest in real applications. These two types of time-frequency structures are typically processed with different classes of methods. In the case of slowly varying structures there are a lot of methods which aim to provide IFL estimation. Time-frequency representations (TFRs) belonging to Cohen's class, adaptive TFRs [2], [3] or parametric TFRs [4] are just few examples of potential methods used for analysis of slowly varying structures. While many drawbacks affect the performing of these methods (cross-terms, computational complexity, needs for a priori information) the polynomial phase modelling arises as an interesting tool for analytical characterization of the IFL
[5], [6]. This type of methods is based on the high-order instantaneous moment (HIM).

In the case of fast varying time-frequency structures the methods cited above are inappropriate. The polynomial phase modelling, for example, cannot be applied since the IFL cannot be approximated by a polynomial. The analysis of fast varying structures is conventionally done by the wavelet-based techniques [7]. That is, numerous works have been done in this field. Therefore, the analysis of both slowly and fast varying structures requires different types of techniques. This can be a serious problem where the processing techniques cannot be arbitrarily complex. The managing of complex techniques and their associated parameters restricts their implementation in real-time applications.

In this paper we extend firstly the HIM concept to deal with phase discontinuities. Namely, by considering complex lags for the computation of the instantaneous moments, we will show that it is possible to characterize phase discontinuities. The results prove that this method is more precise than the wavelet transform. Secondly, the combination of both HIM and complex lag instantaneous moment (CIM) constitutes an efficient tool for the characterization of hybrid IFL (ie composed by slowly and fast varying multi-component T-F structures). Since these methods operate with monodimensional data we will focus on their implementation on a DSP-based architecture.

This paper is organized as follows. In the second paragraph, we present the model of the signals processed by the proposed technique. In this section we focus also on the signals arising from few real applications and we will see that this model corresponds to these signals. In section 3 we will introduce the hybrid method based on the joint use of HIM and CIM. Results for signals illustrated in section 2 are given in section 4 . The real-time implementation skills on a TMS320C6x architecture are discussed in section 5 . The section 6 - "Conclusion" will close this communication.

\section{SIGNAL'S MODEL}

Generally, a signal having an IFL which contains both slowly and fast varying parts can be theoretically written as

$$
s(t)=\exp \left[j 2 \pi \sum_{k} h_{D_{k}}\left(t-\tau_{k}\right) \phi_{k}\left(t-\tau_{k}\right)\right]
$$


where $h$ is the rectangular function of duration $D_{k}, \tau_{k}$ is its origin and $\phi_{k}$ is the instantaneous phase law (IPL) bounded by $\tau_{k}$ and $\tau_{k+1}$. The IPLs $\phi_{k}$ are supposed to be smooth functions and, consequently, they will be used to characterize the slowly varying time-frequency content of the IFL. In this paper, we consider that these functions can be expressed as a fourth order polynomial. This constitutes an appropriate model for a large number of time-frequency slowly varying structure types [5].

The transitory parts of the IPL are represented by the fronts of the rectangular functions $h_{k}$. The estimation of the parameters related to the rectangular function, $\tau_{k}$, provides the localization in time of the fast-varying time-frequency parts.

Expression (1) constitutes a good model for several real-life signals. Let us consider firstly the case of a FSK modulation whose theoretical IFL is displayed in figure 1.

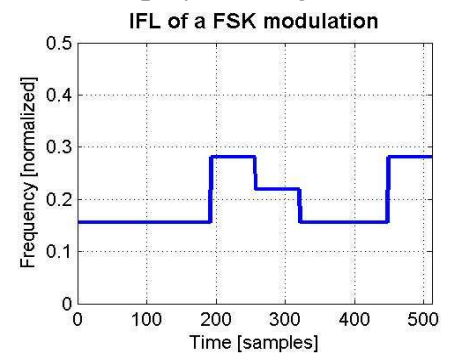

Figure 1. Theoretical IFL of a Frequency Shift Keying (FSK) modulation

This signal can be written, according to (1), as:

$$
\begin{aligned}
& s(t)=\exp j 2 \pi\left[h_{192}(t) \cdot 0.1563 t+h_{64}(t-192) \cdot 0.2813 t+\right. \\
& \left.h_{64}(t-257) \cdot 0.2187 t+h_{130}(t-320) \cdot 0.1563 t+h_{64}(t-450) \cdot 0.2813 t\right]
\end{aligned}
$$

In the second example we consider a signal recorded by an accelerometer connected to a rotating machinery which changes continuously its angular velocity [8]. The theoretical variation of the angular velocity is displayed in the figure 2 .
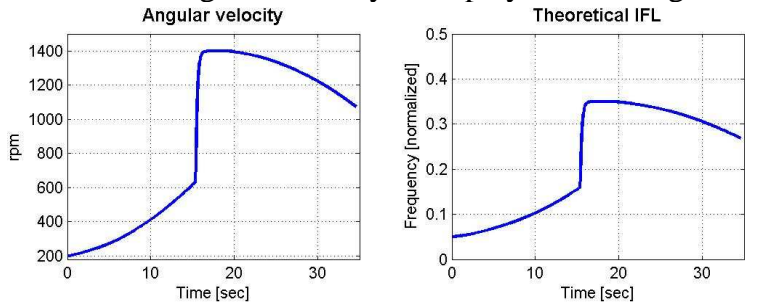

Figure 2. Angular velocity and the corresponding IFL

The angular velocity variation has three phases. In the first phase, between 0 and $16 \mathrm{sec}$, the velocity varies slowly from $200 \mathrm{rpm}$ (rotations per minute) to $610 \mathrm{rpm}$. As it is indicated by the corresponding IFL (the sampling frequency is $8 \mathrm{kHz}$ ) this evolution of the angular velocity is traduced by a slowly varying non-linear time-frequency component. In the second phase, which corresponds to a transition stage, the velocity varies very fast. This could correspond to a man-made changing of the operation conditions. In the domain of the IFL it is associated to a phase discontinuity. Finally, in the last phase, the angular velocity decreases in a non-linear manner as indicated by the IFL as well. According to the model (1) this signal has an IPL characterized by two slowly varying parts and a transient one.

These two examples show that the model (1) can be well matched for real-life signals. The next section will propose a hybrid method which allows the estimation of the parameters of this model.

\section{THE CONCEPT OF INSTANTANEOUS MOMENTS}

\section{A. High-order instantaneous moments}

The High-Order Instantaneous Moments (HIM) given, for a signal $s(t)$, by the following relation :

$$
\left.\left.\operatorname{HIM}_{N}[s(t) ; \tau]=\prod_{q=0}^{\Delta-1}\left[s^{(* q)}(t-q \tau)\right]\right]^{N-1} q\right)
$$

where $N$ is the HIM order, $\tau$ is the lag and $(* q)$ is an operator defined as:

$$
s^{(* q)}(t)=\left\{\begin{array}{cl}
s(t), & \text { if } q \text { is even } \\
s^{*}(t), & \text { if } q \text { is odd }
\end{array}\right.
$$

where $q$ is the number of conjugate operator ' $*$ ' application. From the computational point of view, the main property of the HIM [1] states that the $N^{\text {th }}$-order HIM can be computed as the $2^{\text {nd }}$-order HIM of the $(N-1)^{\text {th }}$-order HIM :

$$
H I M_{N}[s(t) ; \tau]=H I M_{2}\left[H I M_{N-1}[s(t) ; \tau] ; \tau\right]
$$

Another remarkable property, which makes HIM an attractive tool in the polynomial phase modeling context, is that the $N^{\text {th }}$-order HIM of a polynomial phase signal (PPS), defined as $s(t)=\exp \left\{j 2 \pi \sum_{k=1}^{N} a_{k} t^{k}\right\}$ is reduced to a constant amplitude harmonic with amplitude $A^{2^{N-2}}$, frequency $\tilde{\omega}_{N}$ and phase $\tilde{\phi}_{N}[5]$ :

$$
H I M_{N}[s(t) ; \tau]=A^{2^{N-1}} \exp j\left(\tilde{\omega}_{N} \cdot t+\tilde{\phi}_{N}\right)
$$

where :

$$
\begin{aligned}
& \tilde{\omega}_{N}=N ! \tau^{N-1} a_{N} \\
& \tilde{\phi}_{N}=(N-1) ! \tau^{N-1} a_{N-1}-0.5 N !(N-1) \tau^{N} a_{N}
\end{aligned}
$$

A natural idea to take advantage of this property is to compute the Fourier transform of $N^{\text {th }}$-order HIM, which leads to the HAF (High-Order Ambiguity Function) definition [5] :

$$
H A F_{N}[s ; \omega, \tau]=\int_{-\infty}^{\infty} H I M_{N}[s(t) ; \tau] e^{-j \omega t} d t
$$

Obviously, taking into consideration the relation (7), the $N^{\text {th }}$ order HAF peaks at the frequency $\tilde{\omega}_{N}$. This property gives a practical method for polynomial coefficients estimation [5]. Starting with the highest-order coefficient $a_{N}$, the maximum of the HAF is evaluated for each order. The $N^{\text {th }}$-order polynomial coefficient is estimated via : 


$$
\hat{a}_{N}=\frac{1}{N ! \tau^{N-1}} \underset{\omega}{\arg \max }\left\{\left|H A F_{N}(s ; \omega, \tau)\right|\right\}
$$

Using this estimation, the effect of the phase term of the higher order is removed :

$$
s^{(N-1)}(t)=s(t) \cdot e^{-j \hat{a}_{N} t^{N}}
$$

Once the $N^{\text {th }}$-order reduced, the $(N-1)^{\text {th }}$-order HAF is computed. The coefficient $a_{N-1}$ is also estimated thanks to relation (9). The algorithm is iterated through inferior orders until all polynomial coefficients are estimated.

In practice the algorithm based on the HIM operates well for reduced values of highest order $N$. For this reason, the HIM method is inappropriate for analyzing phase discontinuities which would require a high order approximating polynomial.

\section{B. Complex-lag instantaneous moments}

The purpose of the complex lag instantaneous moment is to provide an estimation of the second derivative of the signal's phase. Indeed, this derivative contains very useful information about the phase discontinuities of the signal. For example, the second order derivative of the phase of a FSK signal is defined by Dirac pulses located at the transition points. Therefore, with this kind of information, the detection of the instantaneous frequency transitions is straightforward. A distribution has been designed in [9] to achieve concentration on this derivative which is also called frequency rate. However, this distribution is well suited for polynomial phase signals of order 3 or less. In [10], a complex time distribution was introduced to achieve better accuracy for instantaneous frequency representation. By modifying the complex moment leading to the latter distribution, it is possible to obtain a distribution highly concentrated along frequency rate which is necessary to find points with singular behavior. Thus, we propose the complex lag moment defined by (11).

$$
\begin{aligned}
& C I M[s(t) ; \tau]=s(t+\sqrt{\tau / 2}) \cdot s(t-\sqrt{\tau / 2}) . \\
& s^{*}(t+j \sqrt{\tau / 2}) \cdot s^{*}(t-j \sqrt{\tau / 2})
\end{aligned}
$$

Let suppose that $s(t)$ is of the form (12). The associated distribution (Complex Instantaneous Distribution - CID) is defined by the Fourier transform of (11) and can be written as (13) where $\mathfrak{I}_{t}$ stands for the Fourier transform related to $t$.

$$
\begin{gathered}
s(t)=e^{j \Psi(t)} \\
C I D[\omega, \tau]=\delta\left(\omega-\frac{d^{2} \Psi}{d t^{2}}(t)\right) *{ }_{\omega} \mathfrak{I}_{t}\left(e^{j Q(t, \tau)}\right)
\end{gathered}
$$

One can see that this distribution is spread around the frequency rate. It is more or less spread depending on the value of $Q(t, \tau)$, which would be zero in the ideal case. We give below two different forms of the spreading factor. The first one, (14), is for a distribution similar to [9]. The second one (15) is the spreading factor for our defined distribution.

$$
\begin{aligned}
& Q_{2}(t, \tau)=2 \sum_{k=1}^{+\infty} \Psi^{(2 k+2)}(t) \frac{\tau^{k+1}}{(2 k+2) !} \\
& Q_{4}(t, \tau)=4 \sum_{k=1}^{+\infty} \Psi^{(4 k+2)}(t) \frac{\tau^{2 k+1}}{2^{2 k+1}(4 k+2) !}
\end{aligned}
$$

The first phase derivative implying spreading is of order 6 for our distribution and the next ones are of order $10,14, \ldots$ For the other, the first one is of order 4 and the next ones are of order $6,8,10, \ldots$ Therefore, our distribution is less sensitive to high non-linearities of the signal's phase. Some details related to the implementation can be found in [10].

Both methods presented above are two special cases of instantaneous moment concept. They can be used to analyze the signal modeled by (1). The algorithm issued from the combination of the HIM and CIM is illustrated in the next figure.

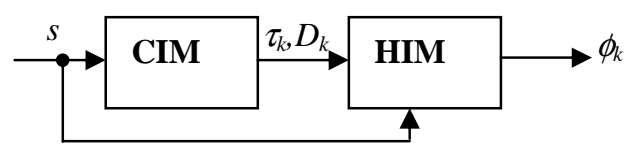

Figure 3 Characterization of signal modeled by (1)

As we can remark on this figure the characterization of the signals according to the model (1) is done in two steps. Firstly, using the CIM methods we estimate the origin of the rectangular windows. Differences between two consecutive values of $\tau_{k}$ lead to the durations $D_{k}$. Secondly, applying the polynomial phase modelling provided by HIM, we estimate the slowly varying parts comprised between to consecutive $\tau_{k}$.

\section{IMPLEMENTATION ISSUES}

In this section we illustrate how the methods presented previously can be implemented in a real-time embedded architecture. As embedded target we consider the TMS320C6711 digital signal processor [11].

Let us take firstly a look on the general implementation architecture of the methods involved in the algorithm devised for the estimation of the model (1). In the case of the HIM, the property expressed by (5) is of a great value for implementation purposes. Namely, it shows that the HIM can be recursively computed with help of of the same HIM operator implemented as indicated in the figure 4.

By applying this operator iteratively it is possible to retrieve all the HIMs of interest. Furthermore, for each HIM, we apply the FFT in order to obtain the associated HAF. The frequency location associated to the maxima of the HAF provides, via (7), the polynomial coefficients.

This algorithm provides the characterization of the timefrequency slowly varying parts of the input signal. 


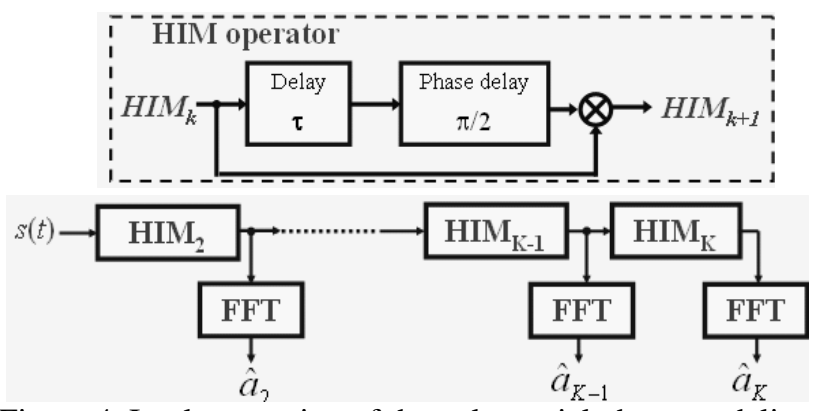

Figure 4. Implementation of the polynomial phase modeling with the HIM
The implementation of the operations implied by the algorithm illustrated in figures 4 and 5 has been done, thanks to their simplicity, with help of the routines available in the libraries associated to the Code Composer Studio (CCS). The programs have been optimized [11] and loaded on the DSP board.

Starting from (11) the implementation of the CIM operator is illustrated in the figure 5 .

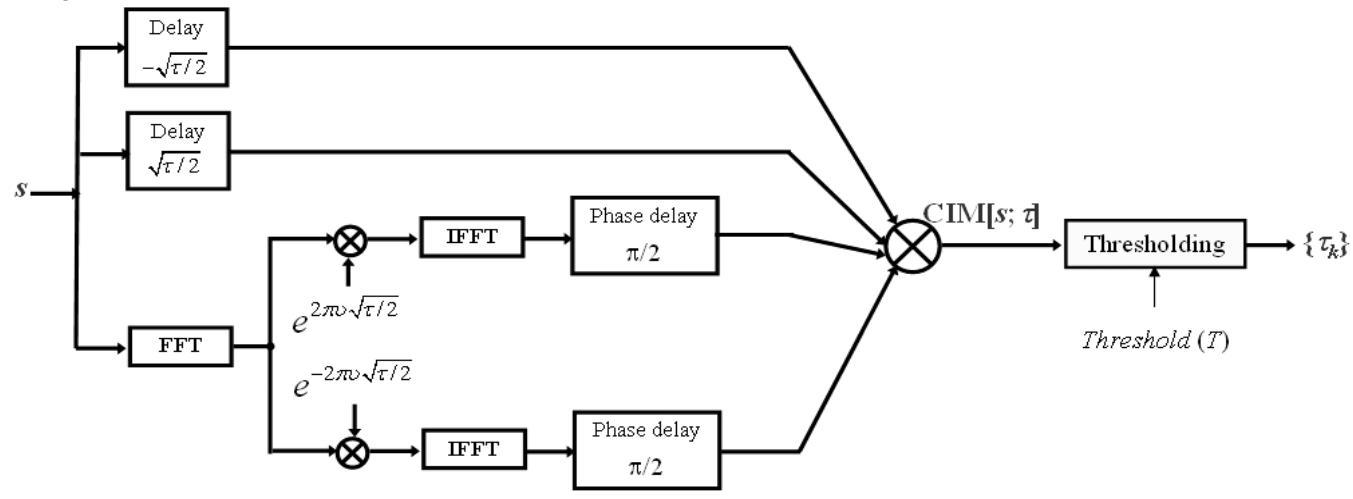

Figure 5. Implementation of the phase discontinuity detection

The general organization of our processing architecture is illustrated in the figure 6 .

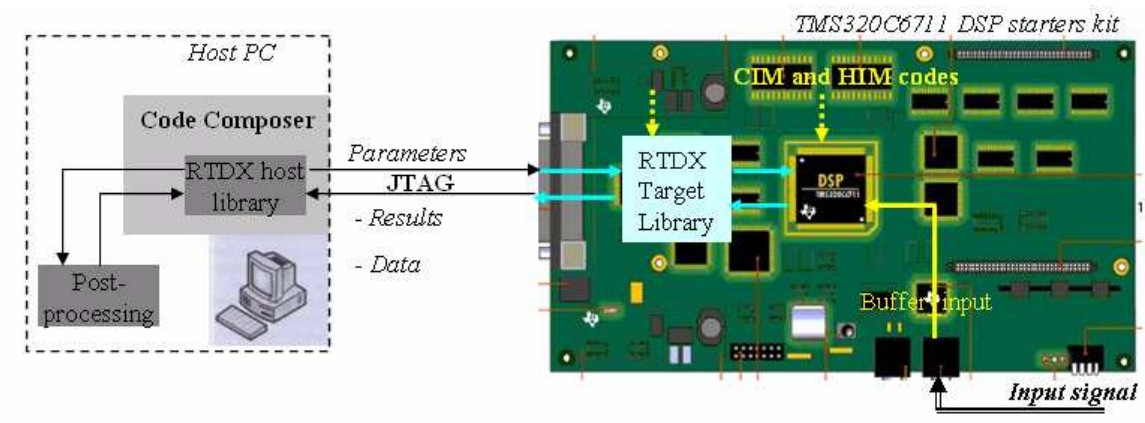

Figure 6. DSP architecture used for the implementation of HIM and CIM processing

The input signal is obtained from the audio coded AD535 at $8 \mathrm{kHz}$ sampling rate [11]. The signal is loaded in a "buffer_input" of 16328 samples size and sent it to the processor. Here, the CIM algorithm is applied in order to detect the time localizations of the phase discontinuities. This detection is based on the CIM (computed according to the figure 5 diagram) thresholding. The time coordinates of the phase discontinuities are loaded in SDRAM memory and they are used to split the "buffer_input" in several temporary buffers containing the signal segmented in time-frequency slowly varying parts. Furthermore, each buffer is sequentially processed by HIM and the polynomial coefficients are also estimated. The polynomial coefficients and phase discontinuities time coordinates are sent to the host PC and the next 16328 samples are introduced in the buffer_input.

During the operation, it is possible to have too much phase discontinuities. In this case, the temporary buffers will be overloaded. In this case, the target will indicate it to the host $\mathrm{PC}$ and the data exceeding the size of the temporary buffers will be transferred and processed to the PC. This situation has not been encountered in our experiments, but in order to be able to tackle it in future applications, we use the RTDX (Real- 
Time Data Exchange) technology to communicate between the $\mathrm{PC}$ and the target. By this way we can continuously exchange data from the PC (parameters required by the methods) and from the target (results and/or signals).

The results are received on the PC and analyzed during the post-processing operation. Otherwise, the data stored on the PC in the case of overloading of the DSP temporal buffers are sent to the target for their processing. Note that in a complete embedded configuration the storing can be done in an external memory board compatible with the DSP.

\section{RESULTS}

In this section we show that the characterization results provided by the proposed methods in the case of signals described in section 2. Both signals have been embedded in a white Gaussian noise with a signal-to-noise ratio equal to 15 $\mathrm{dB}$. For comparison purposes we also tested the Wavelet transform, widely used for the fast varying time-frequency structures and the Wigner-Ville distribution which is the main tool of the Cohen's class.

For the FSK signal the results are plotted in figure 7. As we can observe in the case of the wavelet transform (we plot only the wavelet coefficients issued by high-pass filtering) the frequency steps are visible but the noise introduces artifacts.

Alternatively, the CIM provides only the time localization of the frequency transitions but, compared with the wavelet, this is accurately done. The localization of the transitory parts allows the segmentation of the time-frequency content in slowly varying parts. For each segment, the HIM operator is applied and it provides the polynomial modeling of these parts. For the FSK signal this estimation is plotted by a solid line (figure 7) whereas the theoretical IFL is displayed by a dashed line. We can remark that the estimated parts follow the theoretical IFL. This is due of both accurate estimation of the transient parts and HIM efficiency, respectively. Comparatively, the WVD of the FSK signal is dramatically affected by the cross terms : the time-frequency content is hidden by this phenomenon.
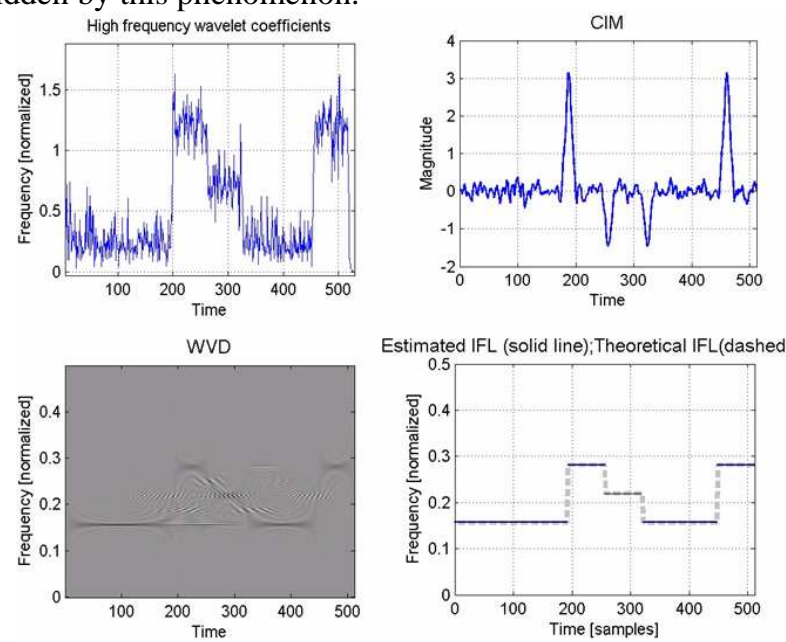

Figure 7. FSK characterization by the proposed method; comparison with wavelet transform and WVD
For the signal issued from the rotating machinery (figure 8) the results proof the efficiency of the method based on joint uses of CIM and HIM. In the case of wavelet transform, the fast transition of the angular velocity is materialized by some energetic wavelet coefficients but, since they are spread over a large time range, the precise localization of the transient part is impossible. Comparatively, the CIM indicates correctly the fast variation of the angular velocity. Concerning the slowly varying parts, contrarily to the WVD, the HIM operator provides an accurate estimation which follows the theoretical
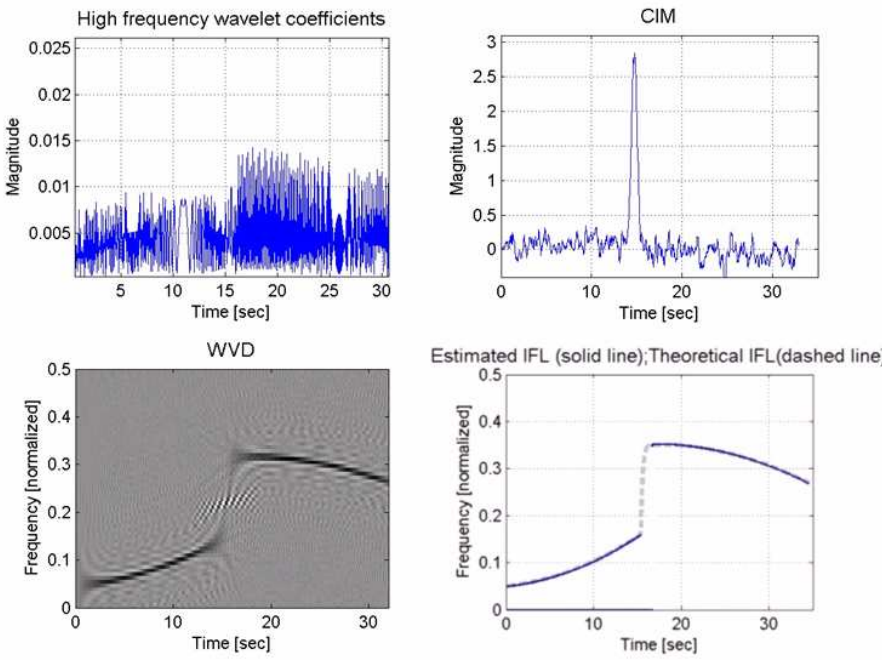

Figure 8. Characterization of a signal issued from a rotating machinery; comparison with wavelet transform and WVD

\section{CONCLUSION}

The main purpose of this paper is to propose a method for the characterization of time-frequency content having both fast and slowly varying structures. This type of content is widely encountered in real-life systems (mechanical, underwater, radar, communication signals, etc) and an efficient characterization of the IFL can provide solutions for problems as classification, physical analysis, monitoring, equalization, etc.

While the conventional analysis of this kind of T-F behavior implies the use of two types of methods (wavelet and Cohen's class distributions, for example) the associated techniques become too sophisticated. Their generalization and implementation could be very complex.

For these reasons we propose a method based on a unique concept - the instantaneous moment. Except its simple structure, well suited for implementation, it leads to two methods - CIM and HIM. The first one constitutes an efficient method for the estimation of time localization of the phase discontinuities. Hence, it provides the segmentation of the signal in slowly varying time-frequency parts. Furthermore, the HIM provides the polynomial characterization of such parts which is a good model for a large variety of real-life signals.

The joint use of CIM and HIM has been tested for two realistic configurations. We pointed out on the main differences between classical methods (wavelet and WVD) and 
the proposed methods. We remarked the efficiency of the proposed approach which accurately characterizes complex time-frequency content.

Taking into account the simplicity of the CIM and HIM definitions, we proposed a real-time embedded structure that implements the proposed approach. This structure is based on a DSP TMS320C6711 and the CIM and HIM methods have been implemented. The results obtained with the DSP have been compared with Matlab simulations and they were similar but the computing time is significantly reduced with the real-time implementation. In addition, the implementation on an embedded structure establishes the base for a future operational embedded system.

From the theoretical point of view, we intend to improve the performances of the existing approach by adding highresolution and multi-component processing capabilities. The application of the proposed approach on real signals will be subject of our future works as well.

\section{ACKNOWLEDGMENT}

This work has been supported by the collaboration program "Pelikan" co-financed by the French and Montenegrin Ministries for Foreign Affairs.

\section{REFERENCES}

[1] A.Papandreou-Suppappola, ed., Applications in time-frequency signal processing, CRC Press, Boca Raton, 2003.

[2] R.G. Baraniuk, D.L. Jones, "A signal-dependent time-frequency representation: fast algorithm for optimal kernel design", IEEE Trans. Signal Processing, 41 (4):1589-1601, April, 1993.

[3] L.J. Stankovic, "L-class of time-frequency distributions", IEEE Signal Processing Letters., vol.3, pp. 22-25, Jan. 1996.

[4] S. Mann, S. Haykin, "The Chirplet Transform : A Generalisation of Gabor's logon", Canadian Image Processing and Pattern Recognition Society, Oct. 1991.

[5] B. Porat, Digital Processing of Random Signals, Pretince Hall, New Jersey, 1993.

[6] S. Barbarossa, A. Scaglione, G.B. Giannakis, "Product High-Order Ambiguity Function for Multicomonent Polynomial-Phase Signal Modeling", IEEE Transactions on Signal Processing, vol. 46, No. 3, March 1998.

[7] S. Mallat, A Wavelet Tour of signal processing, Academic Press, 1998.

[8] W.-X. Yang, X.-M. Ren, "Detecting Impulses in Mechanical Signal by Wavelets", EURASIP Journal of Applied Signal Processing, vol. 8, pp 1156-1162, 2004.

[9] P. O'Shea, "A New Technique for Instantaneous Frequency Rate Estimation", IEEE Signal Processing Letters., vol.9,nº pp. 251252, Aug. 2002.

[10] L.J. Stankovic, "Time-frequency distributions with complex time argument", IEEE Transactions on Signal Processing, Vol. 50, No. 3, pp. 475-486, March 2002.

[11] Texas Instruments, TMS320C6711 Floating Point Digital Signal Processor, 2003. 